Review

\title{
WFDC Protein: A Promising Diagnosis Biomarker of Ovarian Cancer
}

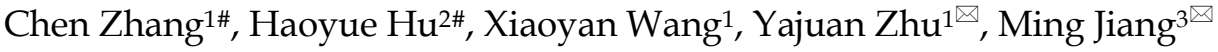 \\ 1. State Key Laboratory of Biotherapy, Cancer Center, West China Hospital, Sichuan University, Chengdu, Sichuan Province, China \\ 2. Lung Cancer Center, Cancer Center, State Key Laboratory of Biotherapy, West China Hospital of Sichuan University, Chengdu, Sichuan, 610041, China \\ 3. West China Hospital, Sichuan University, Chengdu, People's Republic of China \\ \#: These authors contributed equally. \\ $\square$ Corresponding authors: Ming Jiang, West China Hospital, Sichuan University, Chengdu, People’s Republic of China. Email: m18980605799@163.com \\ (C) The author(s). This is an open access article distributed under the terms of the Creative Commons Attribution License (https://creativecommons.org/licenses/by/4.0/). \\ See http://ivyspring.com/terms for full terms and conditions.
}

Received: 2021.01.06; Accepted: 2021.05.23; Published: 2021.07.06

\begin{abstract}
An initial diagnosis of cancer is usually based on symptoms, abnormal physical examination and imaging tests. Ovarian cancer is difficult to be diagnosed timely due to the nonspecific symptoms, thus resulting in the high-risk mortality. Despite of the various diagnostic methods, there is still no reliable diagnostic test. Clinically, carbohydrate antigen $125(\mathrm{CA} 125)$ is widely recognized as a diagnosis biomarker of ovary cancer. However, CA125 is not sensitive to detect the ovary cancer at the early stage. It is essential to explore other potential biomarkers. Human epididymis protein 4 (HE4) in the whey/four-disulfide core (WFDC) proteins family shows satisfactory sensitivity in the early diagnosis of ovary cancer. In this present review, we summarized the important effects of WFDC family proteins on the proliferation, apoptosis and migration of ovary cancer and intended to provide more evidence to explore the possibility of WFDC protein as a diagnosis biomarker.
\end{abstract}

\section{Introduction}

Although ovarian cancer accounts for only a third of gynecologic cancers, it results in $55 \%$ of deaths from gynecologic malignancies and $6 \%$ of all cancer deaths in women. The 50 70-year-old group is a group with the highest incidence of disease [1]. Ovarian cancer with high mortality rate among women has attracted much attention. Although it is not as common as breast cancer, delayed diagnosis may result in distant metastasis and high lethality. Despite advances are being made, ovarian cancer remains the most fatal female gynecologic cancer [2]. Early detection of the disease can significantly improve the cure rate of ovarian cancer. Therefore, the use of biomarkers is extremely important in the diagnosis of ovarian cancer.

In 1980, 'four-disulfide core' (FDC) structure and overall folding patterns have been initially found between snake venom postsynaptic neurotoxin and wheat germ agglutinin domain. This structure is composed of four similar disulfide bonds with different size ring [3]. Then, Hennighausen et al. confirmed that whey acidic protein was the main protein in the milk of rats and mice. The whey-acidic-protein motif in this protein is the prototypic member of a subfamily of the whey/four-disulfide core proteins (WFDC) [4]. The gene encoding proteins of this family are located on human chromosome 20q13. And the proteins are defined by the possession of one or more 40-50 amino acid domains, including eight conserved cysteine residues linked by four characteristic intramolecular disulfide bonds [5]. The proteins of WFDC family have multiple active functions, such as anti-HIV, anti-microbial, immune and cell migration activities [6].

With the emergence of selective molecular targeted therapies, biomarkers play an increasingly crucial role in the clinical management of cancer 
patients [7]. The monitoring of biomarkers can predict the existence, incidence and prognosis of tumors, providing help to clinical treatment and medication guidance. Among various discovered biomarkers, carbohydrate antigen 125 (CA125) is the most commonly used blood-based biomarker for ovarian cancer diagnostic. However, CA125 is elevated in some case of certain common benign diseases, such as endometriosis, follicular cysts, pregnancy and cystadenoma, indicating that CA125 lacks the specificity to predict ovarian cancer [8]. In addition, CA125 detected in the serum of early ovarian cancer patients has quite lower sensitivity than in the advanced stage patients [9]. Thence, it is essential to discover biomarkers that have both high sensitivity and early identification in ovarian cancer. Later, human epididymis protein 4(HE4) protein was found to be highly expressed in ovarian cancer [10]. As a promising biomarker for epithelial ovarian cancer, it shows better specificity and sensitivity in detection [11]. Therefore, it has received widespread attention as a cancer biomarker in recent years. Additionally, the simultaneous measurement of indicators HE4 and CA125 in serum improves the accuracy of ovarian cancer diagnosis and provides the distinction between ovarian tumors and endometriotic cysts [12]. Elevated evidence suggests that WFDC family proteins with whey-acidic-protein (WAP) domain have potential on becoming ranks of biomarker for their diverse functions. We intend to summarize the role of WFDC family with WAP domain as biochemical markers of ovarian cancer.

\section{WFDC family and ovarian cancer}

The attention of researches on cancer has gradually focused on genes coding WFDC family proteins. Most notably, the genes coding for WAP four disulfide core domain protein 14 (WFDC14, also named elafin), WAP four disulfide core domain protein 4 (WFDC4 also named SLPI), WAP four disulphide core domain protein 1 (WFDC1, also named PS20) and WAP four disulphide core domain protein 2 (WFDC2, also named HE4), have been studied on ovarian cancer. In the past ten years, SLPI and HE4 were the most studied molecules of the WFDC family in pathogenesis and development of ovarian cancer [10,13]. In 2010, Elafin was linked with ovarian cancer [14]. Recently, reports on other proteins of WFDC family, such as Elafin and ps20, are gradually increasing [15-17]. These family proteins with WFDC-type protease inhibition activity are encoded by gene localized in chromosome 20q12-q13. It has the frequent amplification region in ovarian cancer, and the WFDC protein family genes located in this chromosome are amplified in several cancers, such as ovarian, breast, colon and prostate cancer. The analyses of data from the Cancer Genome Atlas Program (TCGA) show that HE4, SLPI and elafin all showed significant difference in ovary cancer, while the ps20 proteins expression had no difference (Figure 1). Among these, HE4 has been repeatedly confirmed to have significant expression changes in ovarian cancer tumor. Related basic experiments have proved that HE4 has an impact on tumor progression, such as proliferation, apoptosis invasion and migration [18, 19]. Of note, metastasis and invasion of early-stage ovarian cancer is a major factor responsible for its high mortality and poor prognosis [20]. Elafin, SLPI and ps20 have limited research reports on ovarian cancer. However, these proteins may affect various crucial cancer behaviors through multiple functions (Figure 2), the same as HE4, indicating the crucial role of WFDC family proteins as biomarkers.
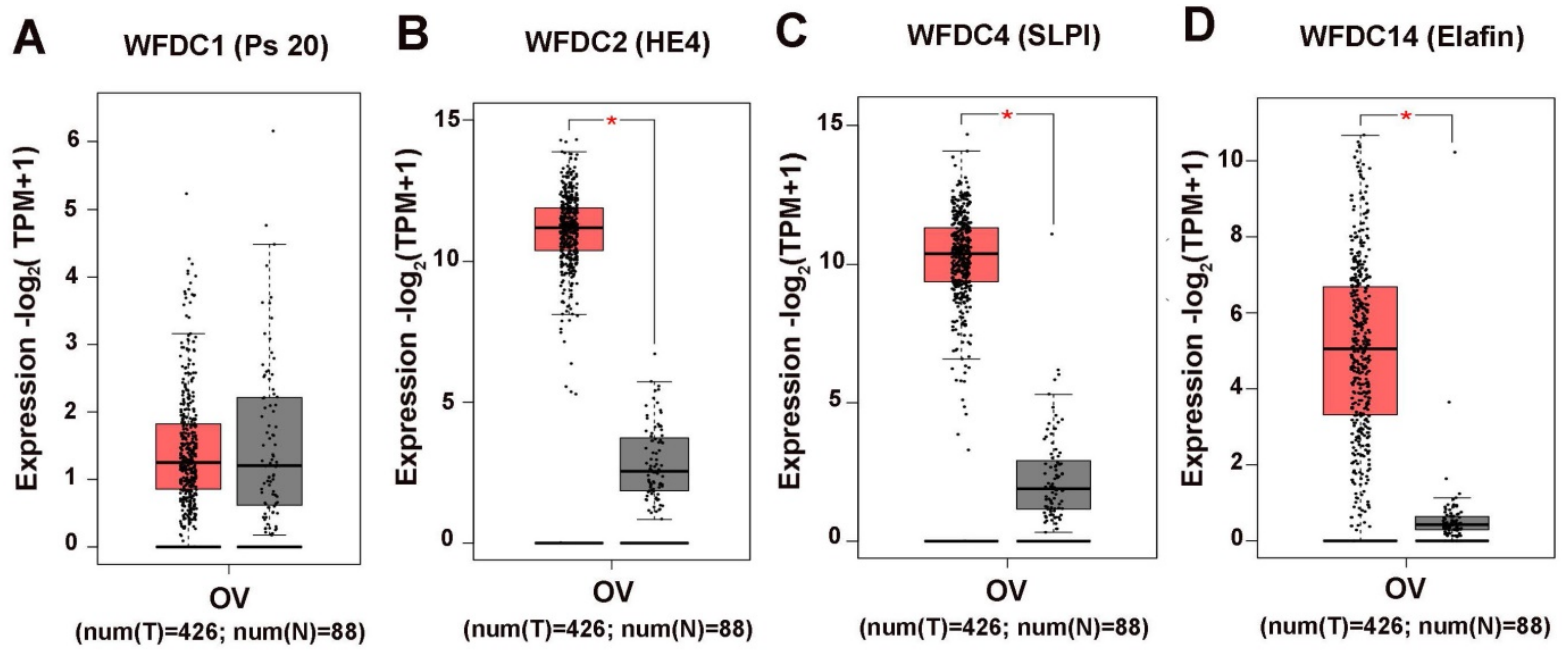

Figure 1. The expression levels of WFDC proteins in ovarian cancer. (A) WFDC1 (B) WFDC2 (C) WFDC4 (D)WFDC14. The red represents the tumor tissue, the gray represents the adjacent tissues, each point represents the expression of the sample $(* P<0.05)$. 


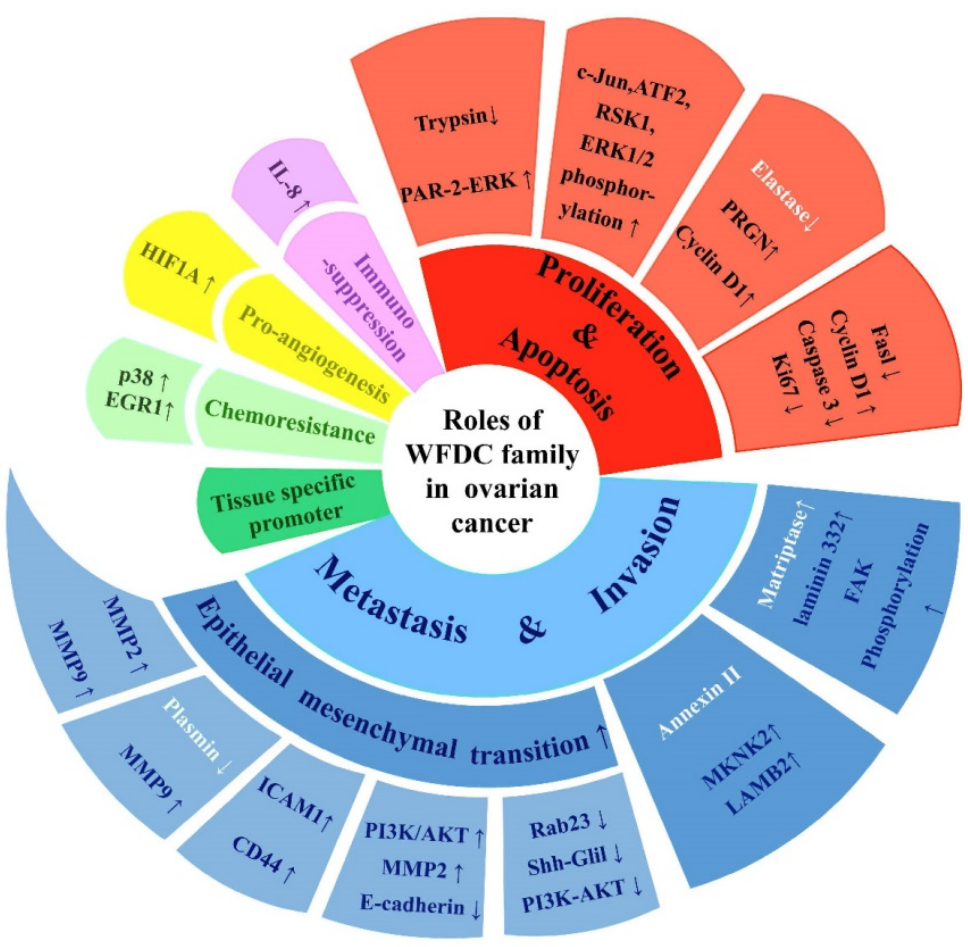

Figure 2. The multiple functions of WFDC family proteins in ovarian cancer. WFDC family proteins have various roles in ovarian cancer, including cell proliferation and apoptosis, migration and invasion, immunosuppression, pro-angiogenesis, chemoresistance, as well as treatment potential as tissue-specific promoters. The white part of the font indicates that the mode of action is not directly through the protease inhibitor activity.

\section{HE4 in ovarian cancer}

HE4 is currently most studied in ovarian cancer. It has been currently evaluated for diagnosing ovarian malignant tumor as a novel biomarker [21]. In the epithelium tissues of respiratory and reproductive organs, HE4 is weakly expressed. On contrast, HE4 is overexpressed in ovarian tumor, especially in endometrioid ovarian cancer [10]. In addition, the overexpression of HE4 can enhance proliferation, apoptosis, invasion and metastasis of ovarian cancer $[19,22]$. A previous study has revealed that silencing of HE4 can lead to impaired capability of human serous ovarian carcinoma cell line SKOV3, such as proliferation, cell cycle, early apoptosis ability of invasion and migration. Meanwhile, it has been confirmed that decrease of these abilities were related to phosphorylated protein of the ERK pathway, JAK-STAT pathway and matrix metalloproteinases $[23,24]$. HE4 as a serine protease inhibitor can also augment the activity of trypsin, thereby enhancing proteinase activated receptor 2 (PAR2)-mediated cell proliferation by ERK pathway [25]. J.R. Ribeiro et al found that HE4 contributes to the collateral resistance to cisplatin and paclitaxel in HE4-overexpression cell lines. The MAPK-regulated response gene involved in promoting metastasis and apoptosis, such as p38 and EGR1, increased [26].

It is interesting that HE4 has an essential effect on tumorigenesis and development without directly exerting its serine protease inhibitory function [27, 28]. In vitro, matriptase activity was enhanced by recombinant HE4 in a dose-dependent manner. It indicated that HE4 can enhance matriptase enzymatic activity of matriptase and, possibly working in concert with upregulation of laminin-332 by HE4 to promote laminin-332 functions, thus affecting migration, invasion, or adhesion. Meanwhile, this study confirmed that HE4 promotes the activation of FAK signaling pathway to stimulate cell adhesion to the extracellular matrix $[29,30]$. Furthermore, there have been several studies reported about the interaction between HE4 and Annexin II (ANXA2) protein by a specific binding model in endometrial carcinoma [31, 32]. As a calcium-dependent phospholipid binding protein annexin ANXA2 II may help HE4 translocate into the nucleus, where it functioned as a transcription factor and increased the expression of MAPK or FOCAL signaling molecules, MAPK interacting serine/threonine kinase 2 (MKNK2) and laminin subunit beta 2 (LAMB2), to promote ovarian cancer cell invasion and metastasis [18]. And HE4 not only regulated the expression of Fasl, cyclin D1, caspase 3 and Ki67 to influence tumor apoptosis and proliferation, but also altered metastasis gene expression such as intercellular adhesion molecule 1 (ICAM-1), CD44, matrix metalloproteinase-2 (MMP2) and matrix metalloproteinase-9 (MMP9) to influence tumor metastasis [22, 24, 33]. The epithelial-mesenchymal 
transition (EMT) process in the progression of cell migration and invasion, as the key point of ovarian metastasis, was influenced by HE4, which promotes the polarization of epithelial cells and imparts mesenchymal cell characteristics. Later, it was proposed that HE4 activated PI3K/AKT signaling pathway and increased the MMP2 expression to influence EMT [34]. The knockdown of HE4 reduced the level of MMP2 and MMP9, further limiting EMT process [24]. In addition, HE4 can upregulate Rab23 protein expression, which also involved in EMT process of ovarian cancer cells by acting Shh-Gli1 and PI3K-AKT signaling pathways [27, 35]. The role of HE4 in affecting metastasis as an expression regulator of EMT is still required further evaluation.

Meanwhile, HE4 contributes to occurrence of inflammation in tumor environment. Recently, it was the first time to confirm that HE4 suppresses STAT3 to upregulate IL8 and HIF1A gene expression. This progress affects microenvironment and angiogenesis of ovarian cancer. Expression of HE4 was associated with reduced $\mathrm{CD}^{+} \mathrm{T}$ cells in tissue of epithelial ovarian cancer patients [36]. Collectively, considering the important role of HE4 in proliferation, metastasis, chemoresistance and immune suppression, HE4 can plausibly be targeted for therapeutic benefit and immunomodulatory effects.

\section{SLPI in ovarian cancer}

SLPI is reported to overexpress in ovarian cancer while it has low expression levels in normal organs $[37,38]$. And upregulation in human ovarian cancer cells was detected upon exposure to paclitaxel, resulting in chemoresistance in part through ERK1/2 activation [39]. Similarly, Elafin can also affect the EMT process, which does not exclusively depend on protease inhibitor activity. The overexpression of SLPI independently on the protease inhibition function is associated with increased proliferation and metastasis of ovarian cancer. SLPI can protect progranulin (PGRN) from proteolysis by binding to the inter-granulin linker areas to block the accessibility to proteases, or inhibiting the converting protease elastase directly [40]. The previous study found that SLPI can protect PRGN from serine protease-mediated degradation. The protective effect of SLPI on PRGN was independent of its protease inhibitory activity and increased cyclin D1 expression proliferation of ovarian cancer [41]. The indirect blocking role is also confirmed by other studies. The protease inhibition-null mutant (PI-/SLPI) can partner with PRGN and provide equal or greater protection for PRGN from elastase [42]. It also has been demonstrated that SLPI had a regulatory direct or indirect effect on MMP quantity through transcriptional upregulation in the nucleus of ovarian cancer cell [43], even though it was lack of a nuclear localization signal. Meanwhile, coupling with its extracellular interaction with plasmin, SLPI inhibited plasmin activity to regulate MMP-9 activation and release. MMP9 is an essential factor of selectively modulating the tumor microenvironment and promoting tumor cell development as an inducer of EMT [44], bringing to light that modulation of activity of SLPI may be therapeutically relevant in ovarian cancer, especially influencing metastasis.

Applying to SLPI as tissue-specific promoter is a potentially useful method for ovarian cancer and allows targeted options for better gene therapy of ovarian cancer. In the ovarian tumor, gene expression regulated by the SLPI promoter was shown to be higher than in normal tissues such as the liver, lung, heart, kidney, and so on. Under the expression control of the SLPI promoter, HSVtk/GCV-mediated cell killing for ovarian cancer considerably improved survival in mice model to a comparable extent as the CMV promoter [45].

\section{Elafin in ovarian cancer}

The first report connecting Elafin to ovarian cancer was presented on 2010. Elafin expression correlated with poor overall survival in late-stage, high-grade serous ovarian carcinomas. Importantly, its expression can be transcriptionally upregulated by immune cytokines via the activation of NF-KB pathway in primary tumors [14]. In high-grade serous ovarian cancer, Elafin overexpressed and was secreted by advanced ovarian cancer, leading a proliferative impact by phosphorylation of c-Jun (S63), ATF2 (S90), ribosomal protein S6 kinase (RSK1) and ERK1/2 through the MEK-ERK pathways [46]. However, another study discovered that Elafin-positive cells were predictors of poor disease-specific survival only in stage I/II ovarian cancer patients. Compared to the normal fallopian tube, Elafin also downregulated in $33 \%$ of ovarian cystadenomas, $43 \%$ of borderline ovarian tumors, and $86 \%$ of invasive ovarian carcinomas [15]. Human epithelial ovarian cells were less sensitive to genotoxic drugs such as cisplatin, carboplatin, cyclophosphamide, and 5-fluorouracil after treatment with Elafin. This effect of cisplatin-resistance caused by Elafin suppressed the cisplatin-induced apoptosis and caspases activation [47]. Despite the fact that Elafin individually is not a highly specific and sensitive diagnostic biomarker for epithelial ovarian cancer now, the monitoring of serum Elafin in combination of CA125 and HE4 may also provide clinically applicable information. 


\section{PS20 in ovarian cancer}

Prostate stromal 20 (ps20) is increasingly recognized as an important regulator of tumor growth, mostly in progressive prostate cancer [48, 49]. Previous researches have mostly explored how ps20 might impact prostate cancer behaviors $[49,50]$. The latest reports highlighted that sorbin and $\mathrm{SH} 3$ domain containing 2 (SORBS2) binds the 3' untranslated regions (UTRs) of ps20 to enhance the stability of these gene transcripts, which suppresses the invasiveness of ovarian cancer [17]. In addition, ps20 expression was shown to be substantially lower in metastatic ovarian cancer sites as compared to original sites by examining the Oncomine database. Furthermore, there was positive correlation between ps20 expression with overall survival of ovarian cancer patients [17]. It has been shown that ps20 may be intimately linked to disease migration WFDC protein as a biomarker in the detection of early-stage ovarian cancer seems promising, according to further in-depth mechanistic study.

\section{WFDC protein can be recognized as promising biomarker of diagnosis and treatment in ovarian cancer}

In comparison to other cancers associated with women, $77 \%$ of them are diagnosed as endometrial cancers stage I, $55 \%$ of breast cancers and $83 \%$ of cervical cancers respectively. While only $23 \%$ of ovarian malignancies are detected at an early stage [51]. Therefore, there are no clear indicators that can be utilized in clinic. The early diagnosis of ovarian cancer is continuously being researched. Successful screening of ovarian cancer is necessary. The CA125 was firstly described in the early 1980's. The identification of CA125 as a circulating antigen paved the way for ovarian carcinoma biomarker research [52]. Then, several various tumor biomarkers have been evaluated via laboratory tests (Table 1).

CA125 is originally used to monitor the chemotherapy response of ovarian cancer and detect the relapse after surgery [53], which is a peptide epitope of 3-5 million Da mucin (MUC16) [54]. MUC16, commonly known as CA125, is not only a biomarker for ovarian cancer, but also evolves in tumor progression and metastasis [55]. Due to their aberrant overexpression and functional involvement, MUC16 and its ligands have emerged as prospective targets for therapeutic intervention utilizing monoclonal antibodies and immunotherapy $[56,57]$. However, cleavage of extracellular MUC16 domain, an inadequate understanding structural variety of the MUC16 domains and uncharacterized associated signaling pathway under illness circumstances all provide obstacles to the therapeutic development of MUC16 or CA125 [57]. Recently, levels of CA125 have recently been universally acknowledged as increasing in other several benign conditions. CA125 elevates in $80 \%$ of patients with epithelial ovarian cancer at initial diagnosis and correlates strongly with response to therapy, while it has extremely limited sensitivity in identifying patients in early-stage ovarian cancer [58].

In a randomized controlled trial, annual multimodal screening with serum CA125 and transvaginal ultrasound sonography as a second-line diagnostic demonstrated minimal effectiveness [59]. A recent research has suggested that discovering biomarkers in urine can provide a non-invasive technique for detecting ovarian cancer, and allow the frequent testing of women with high-risk. In contrast, CA125, a typical serum tumor biomarker in ovarian cancer, showed no significance in the urine data [60].

Secretory HE4 showed significant difference as urinary biomarker in the differential diagnosis between ovarian cancer and benign tumors, followed by creatinine, carcinoembryonic antigen, vascular cell adhesion molecule (VCAM) and transthyretin (TTR) [60]. The HE4 expression levels in serum and urine of ovarian cancer patients was significantly upregulated compared with normal or benign groups [24]. HE4 as a biomarker in early-stage cancer was not overexpressed in benign ovarian disease, normal ovarian tissue or tumors with low malignant potential [61]. Meta-analysis from multiple studies systematically evaluated the potential of urine HE4 as a non-invasive biomarker for the diagnosis of ovarian cancer [62]. In this meta-analysis, four Asian studies had explicitly evaluated the diagnostic value of urine HE4 and serum HE4, demonstrating that urine HE4 had a higher pooled sensitivity (0.82 vs. 0.74$)$ and a higher pooled specificity (0.93 vs 0.88 ) compared to serum HE4. The area under the curve (AUC) of summary receiver operating characteristic (SROC) for urine HE4 and serum HE4 were 0.92 and 0.95, respectively, which indicated that urine HE4 may be superior to serum HE4 in screening ovarian cancer. Earlier research indicated that compared to concentration of serum HE4, urinary concentrations of HE4 elevated in patients with early and advanced ovarian cancer, as well as in patients with serous ovarian cancer [63], which uncovered that urinary HE4 may be more sensitive than serum HE4 analysis in certain cases of ovarian cancer. The protein profile in urine is less complicated than that in blood, thus overall clinical performance is enhanced by the measurement of urinary proteins. Meanwhile, certain proteins may be more stable in urine than in blood. Moreover, urinary tests would be more convenient than invasive blood tests. HE4, with a molecular 
weight of $25 \mathrm{kDa}$, is below the glomerular filtration cutoff [64]. WFDC family including HE4 is composed of small secretory molecular protein, so it may be worth expanding areas for urine biomarker to detect early-stage ovarian cancer. Simultaneously, it has potential to create novel methods of ovarian cancer monitoring, such as monitoring through urine while enhancing specificity and sensitivity.

Diagnostic comparisons of potential single biomarker, including HE4, CA125, carcinoembryonic antigen (CEA), carbohydrate antigen 19-9 (CA19-9), transthyretin (TTR) and apolipoprotein A-1 (ApoA-1), also indicate HE4 has important roles in diagnosing [65-67], particularly in early-stage (Table 1). The serum CA125 and computed tomography (CT) imaging are currently used for evaluating primary therapy response evaluation in epithelial ovarian cancer [68], while the postoperative CA125 level is an unreliable indicator for residual tumor. Instead, postoperative HE4 is statistically significantly associated to residual tumor and first line chemotherapy after primary debulking surgery and interval debulking surgery in patients with advanced epidermal ovarian carcinoma [69]. Several clinical investigations assess the sensitivity and specificity of HE4 and CA125 indicators. Unlike CA125, serum levels of HE4 are not elevated in common benign conditions, such as pregnancy and endometriosis. CA125 has higher sensitivity than HE4 in both premenopausal and postmenopausal women, while HE4 has the highest specificity in both menstrual states [70-72] (Table 1). Several clinical trials have noted that HE4 levels had equal sensitivity but higher specificity in detecting ovarian cancer when compared to CA125 levels [72, 73]. SLPI, which belongs to the same family as HE4, has been shown to be an excellent predictor of epidermal ovarian cancer [74]. Currently, HE4 protein is still the most researched protein of WFDC family which is used for ovarian cancer biomarker in clinical trials. Efficacy of other WFDC family proteins needs further researched by clinical trial.

Advances in biomarker discovery have led to several FDA-approved tests superior to CA125 in preoperative evaluation of women with a pelvic mass, including ROMA, Overa and OVA1 test (Table 2). It is an initial enthusiasm to estimate the possibility of malignancy as a triage to ovarian masses and diagnostic aid, although they are not ideal diagnostic tests for early-stage detection. It presents a trend of variations that exploring biomarkers alternatives to CA125 alone and complements diagnostic performance in early-stage. A new insight of combination of markers to detect the occurrence and development of early ovarian cancer may improve the sensitivity and accuracy of detection (Table 2). Multiple biomarker panels, including HE4 protein, are being studied in depth [75, 76]. Combined measurements HE4 and CA125 can also be useful in differential diagnosis of different pathological type of ovarian cancer, especially diagnosis between ovarian endometrioma and epithelial ovarian cancer [77]. Studies using the combined biomarkers in ovarian cancer patients are warranted. And it has great potential in exploring new biomarkers complemented to the early diagnostic.

Table 1. Comparison of diagnostic performance of multiple biomarker alone in clinical analysis.

\begin{tabular}{|c|c|c|c|c|c|c|c|c|c|c|}
\hline Biomarker & Subject & Grouping & Physiological Condition & $\mathrm{SN}(\%)$ & $\mathrm{SP}(\%)$ & PPV(\%) & NPV $(\%)$ & ROC & Cutoff & Reference \\
\hline \multirow[t]{7}{*}{ CA125 } & 419 & EOC:114/27.2\% & PreM & 92.3 & 59.4 & - & - & - & $35 \mathrm{U} / \mathrm{ml}$ & {$[78]$} \\
\hline & & & PostM & 94.3 & 82.3 & - & _- & _- & & \\
\hline & 373 & Malignancy:55/14.7\% & PreM & 85.7 & 55.7 & - & _- & _- & $35 \mathrm{U} / \mathrm{ml}$ & [79] \\
\hline & & & PostM & 88.1 & 85.0 & - & - & - & & \\
\hline & 458 & Malignancy:196/42.8\% & & 62.8 & 70.6 & _ & _ & 0.717 & $35 \mathrm{U} / \mathrm{ml}$ & {$[80]$} \\
\hline & 263 & OC: $118 / 44.9 \%$ & stage I \& II & 51.6 & 51.2 & - & - & - & $35 \mathrm{U} / \mathrm{ml}$ & [81] \\
\hline & & & stage III \& IV & 94.3 & 84.3 & - & - & - & & \\
\hline \multirow[t]{5}{*}{ HE4 } & 419 & EOC:114/27.2\% & PreM & 84.6 & 94.2 & - & - & - & $70 \mathrm{pM} / \mathrm{L}$ & [78] \\
\hline & & & PostM & 78.2 & 99.0 & - & _ & _- & $140 \mathrm{pM} / \mathrm{L}$ & \\
\hline & 373 & Malignancy:55/14.7\% & PreM & 57.1 & 100.0 & - & - & _- & $140 \mathrm{pM} / \mathrm{L}$ & [79] \\
\hline & & & PostM & 71.2 & 10.0 & _ & _ & - & & \\
\hline & 458 & Malignancy:196/42.8\% & - & 63.8 & 96.6 & - & - & 0.967 & $140 \mathrm{pM} / \mathrm{L}$ & {$[80]$} \\
\hline \multirow[t]{2}{*}{ CEA } & 458 & Malignancy:196/42.8\% & - & 38.8 & 88.6 & _- & - & 0.777 & $5 \mathrm{ng} / \mathrm{ml}$ & {$[80]$} \\
\hline & 266 & OC: $19 / 7.1 \%$ & - & 11.1 & & 11.2 & 95.0 & - & $4.6 \mathrm{ng} / \mathrm{ml}$ & [82] \\
\hline \multirow[t]{2}{*}{ CA19-9 } & 458 & Malignancy:196/42.8\% & - & 35.7 & 79.0 & - & - & 0.624 & $27 \mathrm{U} / \mathrm{ml}$ & {$[80]$} \\
\hline & 266 & OC: $19 / 7.1 \%$ & - & 16.7 & - & 15.8 & 95.4 & - & $43 \mathrm{kIU} / \mathrm{L}$ & [82] \\
\hline \multirow[t]{4}{*}{ TTR } & 256 & OC:126/49.2\% & stage I \& II & 78.6 & - & 68.8 & - & 0.912 & - & [83] \\
\hline & & & stage III \& IV & 82.9 & - & 74.3 & - & 0.932 & - & \\
\hline & 263 & OC:118/44.9\% & stage I \& II & 61.2 & 59.2 & - & - & - & $100 \mathrm{ng} / \mathrm{ml}$ & [81] \\
\hline & & & stage III \& IV & 74.9 & 68.2 & _ & _ & _ & & \\
\hline \multirow[t]{2}{*}{ ApoA-1 } & 263 & OC:118/44.9\% & stage I \& II & 51.3 & 54.6 & - & - & - & $500 \mathrm{ng} / \mathrm{ml}$ & {$[81]$} \\
\hline & & & stage III \& IV & 58.4 & 55.1 & _ & _ & _ & & \\
\hline
\end{tabular}

CEA: carcinoembryonic antigen; CA19-9: Carbohydrate antigen 19-9; TTR: Transthyretin; ApoA-1: Apolipoprotein A-1.

EOC: Epithelial ovarian cancer; OC: Ovarian cancer; SN: Sensitivity; SP: Specificity; PPV: Positive predictive value; NPV: Negative predictive value; ROC: Receiver operator characteristic. 
Table 2. Comparison of diagnostic performance of multimarker test in clinical analysis.

\begin{tabular}{|c|c|c|c|c|c|c|c|c|c|}
\hline $\begin{array}{l}\text { Multimarker } \\
\text { Test }\end{array}$ & Subject & Grouping & $\begin{array}{l}\text { Physiological } \\
\text { Condition }\end{array}$ & $\mathrm{SN}(\%)$ & $\mathrm{SP}(\%)$ & PPV & NPV & Cutoff & Reference \\
\hline CA125+HE4+ & 1097 & OC:168/15.3\% & stage I \& II & 86 & $98 \%$ & - & - & - & {$[84]$} \\
\hline CEA+VCAM-1 & & & stage III \& IV & 95 & & _- & _- & _ & \\
\hline CA125+HE4+E-CAD+IL-6 & 171 & OC:125/73.1\% & - & 84.2 & 95.7 & 88.9 & 93.6 & _ & [76] \\
\hline \multirow[t]{7}{*}{$\mathrm{ROMA}+$} & 419 & EOC:114/27.2\% & PreM & 84.6 & 81.2 & - & - & preM 7.4\%; & {$[78]$} \\
\hline & & & PostM & 93.1 & 84.4 & - & - & postM $25.3 \%$ & \\
\hline & 472 & EOC: $48 / 10.2 \%$ & PreM & 100 & 74.2 & 17.8 & 97.4 & preM $13.1 \%$; & [85] \\
\hline & & & PostM & 92.3 & 76 & 56.1 & 100 & postM $27.7 \%$ & \\
\hline & 373 & Malignancy:55/14.7\% & PreM & 72.5 & 79.1 & - & - & preMindex11.4; & [79] \\
\hline & & & PostM & 87.3 & 91.7 & _- & - & postM index 29.9 & \\
\hline & 458 & Malignancy:196/42.8\% & - & 81.1 & 84 & - & - & - & [80] \\
\hline OVA1++ & 493 & Malignancy:92/18.7\%\% & - & 94.2 & 53.5 & 31.4 & 96.8 & - & [86] \\
\hline \multirow[t]{3}{*}{ Overa* } & 493 & Malignancy:92/18.7\%\% & - & 91.3 & 69.1 & 40.4 & 97.2 & - & [86] \\
\hline & 218 & EOC: $64 / 29.4 \%$ & PreM & 78.3 & 72.3 & 58.1 & 87.2 & - & [87] \\
\hline & & & Post M & 90.1 & 62.3 & 68.8 & 87.3 & _ & \\
\hline
\end{tabular}

+, Roma Algorithm;

*, CA125, HE4, transferrin (TRF), apolipoprotein A-1 (ApoA-1) and follicle-stimulating hormone (FSH).

,++ CA 125, transferrin (TRF), transthyretin (TTR), apolipoprotein A1 (ApoA1), and $\beta 2$-microglobulin (B2M);

EOC: Epithelial ovarian cancer; OC: Ovarian cancer; SN: Sensitivity; SP: Specificity; PPV: Positive predictive value; NPV: Negative predictive value.

\section{Summary and Prospects}

WFDC family proteins are involved in several aspects of the occurrence and development of ovarian cancer, including proliferation, apoptosis, invasion and metastasis. HE4 Ovarian Cancer Monitoring Trial set up to measure the HE4 protein level in blood for the diagnosis. Basic researches and clinical trials are expected to investigate the efficacy of other molecules of WFDC family compounds as biomarker in monitoring, diagnosis and treatment of ovarian cancer, especially in early-stage. We anticipate that further studies on the sensitivity and specificity of molecules other than HE4 in clinical trials will be undertaken to aid in the diagnosis, treatment and prognostic monitoring of early-stage ovarian cancer.

\section{Abbreviations}

CA125: carbohydrate antigen 125; HE4: human epididymis protein 4; WFDC: whey/four-disulfide core; WFDC1: WAP four disulfide core domain protein 1; WFDC2: WAP four disulfide core domain protein2; WFDC4: WAP four disulfide core domain protein 4; WFDC14: WAP four disulfide core domain protein 14; PAR2: proteinase activated receptor 2; ANXA2: annexin II; EMT: epithelial mesenchymal transition; MKNK2: MAPK interacting serine/ threonine kinase 2; Ps20: prostate stromal 20; LAMB2: laminin subunit beta 2 ; ICAM-1: intercellular adhesion molecule 1; MMP2: matrix metalloproteinase 2; MMP9: matrix metalloproteinase 9; PRGN: protect progranulin; RSK1: ribosomal S6 kinase 1; SORBS2: sorbin and SH3 domain containing 2; UTRs: untranslated regions; VCAM: vascular cell adhesion molecule; TTR: transthyretin; AUC: area under the curve; SROC: summary receiver operating characteristic; CA19-9: carbohydrate antigen 19-9; ApoA-1: apolipoprotein A-1.

\section{Author Contributions}

Chen Zhang wrote the original draft. Haoyue $\mathrm{Hu}$ revised the manuscript. Xiaoyan Wang summarized the literature. Yajuan Zhu provided an overall idea. Ming Jiang modified the framework and re-designed pictures and tables.

\section{Competing Interests}

The authors have declared that no competing interest exists.

\section{References}

1. Cancer Research UK. 2014. www.cancerresearchuk.org/cancer-info/ cancerstats/types/ovary/incidence/uk-ovarian-cancer-incidence-statistics.

2. Jayson GC, Kohn EC, Kitchener HC, Ledermann JA. Ovarian cancer. Lancet. 2014; 384(9951): 1376-88.

3. Drenth J, Low BW, Richardson JS, Wright CS. The toxin-agglutinin fold. A new group of small protein structures organized around a four-disulfide core. J Biol Chem. 1980; 255(7): 2652-5.

4. Hennighausen LG, Sippel AE. Mouse whey acidic protein is a novel member of the family of 'four-disulfide core' proteins. Nucleic Acids Res. 1982; 10(8): 2677-84.

5. Chhikara N, Saraswat M, Tomar AK, Dey S, Singh S, Yadav S. Human epididymis protein-4 (HE-4): a novel cross-class protease inhibitor. PLoS One. 2012; 7(11): e47672.

6. Small DM, Doherty DF, Dougan CM, Weldon S, Taggart CC. The role of whey acidic protein four-disulfide-core proteins in respiratory health and disease. Biol Chem. 2017; 398(4): 425-40.

7. Goossens N, Nakagawa S, Sun X, Hoshida Y. Cancer biomarker discovery and validation. Transl Cancer Res. 2015; 4(3): 256-69.

8. Kulbe H, Otto R, Darb-Esfahani S, Lammert H, Abobaker S, Welsch G, et al. Discovery and validation of novel biomarkers for detection of epithelial ovarian cancer. Cells. 2019; 8(7):713.

9. Urban N, McIntosh MW, Andersen M, Karlan BY. Ovarian cancer screening. Hematol Oncol Clin North Am. 2003; 17(4): 989-1005, ix.

10. Drapkin R, von Horsten HH, Lin Y, Mok SC, Crum CP, Welch WR, et al. Human epididymis protein 4 (HE4) is a secreted glycoprotein that is overexpressed by serous and endometrioid ovarian carcinomas. Cancer Res. 2005; 65(6): 2162-9.

11. Lahlou N, Brun JL. [Ovarian tumor markers of presumed benign ovarian tumors]. J Gynecol Obstet Biol Reprod (Paris). 2013; 42(8): 760-73.

12. Nolen B, Velikokhatnaya L, Marrangoni A, De Geest K, Lomakin A, Bast RC $\mathrm{Jr}$, et al. Serum biomarker panels for the discrimination of benign from malignant cases in patients with an adnexal mass. Gynecol Oncol. 2010; 117(3): $440-5$.

13. Tsukishiro S, Suzumori N, Nishikawa H, Arakawa A, Suzumori K. Use of serum secretory leukocyte protease inhibitor levels in patients to improve specificity of ovarian cancer diagnosis. Gynecol Oncol. 2005; 96(2): 516-9.

14. Clauss A, Ng V, Liu J, Piao H, Russo M, Vena N, et al. Overexpression of elafin in ovarian carcinoma is driven by genomic gains and activation of the nuclear 
factor kappaB pathway and is associated with poor overall survival. Neoplasia. 2010; 12(2): 161-72.

15. Caruso JA, Karakas C, Zhang J, Yi M, Albarracin C, Sahin A, et al. Elafin is downregulated during breast and ovarian tumorigenesis but its residual expression predicts recurrence. Breast Cancer Res. 2014; 16(6): 3417.

16. Song H, Kwan SY, Izaguirre DI, Zu Z, Tsang YT, Tung CS, et al. PAX2 expression in ovarian cancer. Int J Mol Sci. 2013; 14(3): 6090-105.

17. Zhao L, Wang $\mathrm{W}$, Huang $\mathrm{S}$, Yang $\mathrm{Z}, \mathrm{Xu} \mathrm{L}$, Yang Q, et al. The RNA binding protein SORBS2 suppresses metastatic colonization of ovarian cancer by stabilizing tumor-suppressive immunomodulatory transcripts. Genome Biol. 2018; 19(1): 35.

18. Zhuang H, Tan M, Liu J, Hu Z, Liu D, Gao J, et al. Human epididymis protein 4 in association with Annexin II promotes invasion and metastasis of ovarian cancer cells. Mol Cancer. 2014; 13: 243

19. Gao L, Cheng HY, Dong L, Ye X, Liu YN, Chang XH, et al. The role of HE4 in ovarian cancer: inhibiting tumour cell proliferation and metastasis. J Int Med Res. 2011; 39(5): 1645-60.

20. Lengyel E. Ovarian cancer development and metastasis. Am J Pathol. 2010; 177(3): 1053-64.

21. Hellstrom I, Raycraft J, Hayden-Ledbetter M, Ledbetter JA, Schummer M, McIntosh M, et al. The HE4 (WFDC2) protein is a biomarker for ovarian carcinoma. Cancer Res. 2003; 63(13): 3695-700.

22. Chen $\mathrm{Y}, \mathrm{Mu} \mathrm{X}$, Wang S, Zhao L, Wu Y, Li J, et al. WAP four-disulfide core domain protein 2 mediates the proliferation of human ovarian cancer cells through the regulation of growth- and apoptosis-associated genes. Oncol Rep. 2013; 29(1): 288-96

23. Zhu YF, Gao GL, Tang SB, Zhang ZD, Huang QS. Effect of WFDC 2 silencing on the proliferation, motility and invasion of human serous ovarian cancer cells in vitro. Asian Pac J Trop Med. 2013; 6(4): 265-72.

24. Wang A, Jin C, Tian X, Wang Y, Li H. Knockdown of HE4 suppresses aggressive cell growth and malignant progression of ovarian cancer by inhibiting the JAK/STAT3 pathway. Biol Open. 2019; 8(9):bio043570.

25. Kim KK, Turner R, Khazan N, Kodza A, Jones A, Singh RK, et al. Role of trypsin and protease-activated receptor-2 in ovarian cancer. PLoS One. 2020; 15(5): e0232253.

26. Ribeiro JR, Schorl C, Yano N, Romano N, Kim KK, Singh RK, et al. HE4 promotes collateral resistance to cisplatin and paclitaxel in ovarian cancer cells. J Ovarian Res. 2016; 9(1): 28.

27. Zhu L, Zhuang H, Wang H, Tan M, Schwab CL, Deng L, et al. Overexpression of HE4 (human epididymis protein 4) enhances proliferation, invasion and metastasis of ovarian cancer. Oncotarget. 2016; 7(1): 729-44.

28. Lu R, Sun X, Xiao R, Zhou L, Gao X, Guo L. Human epididymis protein 4 (HE4) plays a key role in ovarian cancer cell adhesion and motility. Biochem Biophys Res Commun. 2012; 419(2): 274-80.

29. Zhao J, Guan JL. Signal transduction by focal adhesion kinase in cancer. Cancer Metastasis Rev. 2009; 28(1-2): 35-49.

30. Ribeiro JR, Gaudet HM, Khan M, Schorl C, James NE, Oliver MT, et al. Human epididymis protein 4 promotes events associated with metastatic ovarian cancer via regulation of the extracelluar matrix. Front Oncol. 2017; 7: 332

31. Deng L, Gao Y, Li X, Cai M, Wang H, Zhuang H, et al. Expression and clinical significance of annexin A2 and human epididymis protein 4 in endometrial carcinoma. J Exp Clin Cancer Res. 2015; 34(1): 96.

32. Zhuang H, Tan M, Liu J, Li X, Gao J, Hu Z, et al. The expression of annexin II and Lewis $y$ antigen in ovarian epithelial tumors and the correlation between them. Tumour Biol. 2015; 36(4): 2343-9.

33. Chen Y, Huang L, Wang S, Liu T, Wu Y, Li JL, et al. WAP four-disulfide core domain protein 2 promotes metastasis of human ovarian cancer by regulation of metastasis-associated genes. J Ovarian Res. 2017; 10(1): 40.

34. Chen $\mathrm{Y}$, Huang $\mathrm{L}$, Wang $\mathrm{S}, \mathrm{Li} J \mathrm{~L}, \mathrm{Li} \mathrm{M}, \mathrm{Wu} \mathrm{Y}$, et al. WFDC2 contributes to epithelial-mesenchymal transition (EMT) by activating AKT signaling pathway and regulating MMP-2 expression. Cancer Manag Res. 2019; 11: 2415-24

35. Gao L, Zheng M, Guo Q, Nie X, Li X, Hao Y, et al. Downregulation of Rab23 inhibits proliferation, invasion, and metastasis of human ovarian cancer. Int J Biochem Cell Biol. 2019; 116: 105617.

36. James NE, Emerson JB, Borgstadt AD, Beffa L, Oliver MT, Hovanesian V, et al. The biomarker HE4 (WFDC2) promotes a pro-angiogenic and immunosuppressive tumor microenvironment via regulation of STAT3 target genes. Sci Rep. 2020; 10(1): 8558.

37. Hough CD, Cho KR, Zonderman AB, Schwartz DR, Morin PJ. Coordinately up-regulated genes in ovarian cancer. Cancer Res. 2001; 61(10): 3869-76.

38. Abe $\mathrm{T}$, Tominaga $\mathrm{Y}$, Kikuchi $\mathrm{T}$, Watanabe $\mathrm{A}$, Satoh $\mathrm{K}$, Watanabe $\mathrm{Y}$, et al. Bacterial pneumonia causes augmented expression of the secretory leukoprotease inhibitor gene in the murine lung. Am J Respir Crit Care Med. 1997; 156(4 Pt 1): 1235-40.

39. Rasool N, LaRochelle W, Zhong H, Ara G, Cohen J, Kohn EC. Secretory leukocyte protease inhibitor antagonizes paclitaxel in ovarian cancer cells. Clin Cancer Res. 2010; 16(2): 600-9.

40. Arechavaleta-Velasco F, Perez-Juarez CE, Gerton GL, Diaz-Cueto L. Progranulin and its biological effects in cancer. Med Oncol. 2017; 34(12): 194.

41. Simpkins FA, Devoogdt NM, Rasool N, Tchabo NE, Alejandro EU, Kamrava $\mathrm{MM}$, et al. The alarm anti-protease, secretory leukocyte protease inhibitor, is a proliferation and survival factor for ovarian cancer cells. Carcinogenesis. 2008; 29(3): 466-72.
42. Devoogdt N, Rasool N, Hoskins E, Simpkins F, Tchabo N, Kohn EC. Overexpression of protease inhibitor-dead secretory leukocyte protease nhibitor causes more aggressive ovarian cancer in vitro and in vivo. Cancer Sci. 2009; 100(3): 434-40.

43. Hoskins E, Rodriguez-Canales J, Hewitt SM, Elmasri W, Han J, Han S, et al. Paracrine SLPI secretion upregulates MMP-9 transcription and secretion in ovarian cancer cells. Gynecol Oncol. 2011; 122(3): 656-62.

44. Lee YH, Albig AR, Regner M, Schiemann BJ, Schiemann WP. Fibulin-5 initiates epithelial-mesenchymal transition (EMT) and enhances EMT induced by TGF-beta in mammary epithelial cells via a MMP-dependent mechanism. Carcinogenesis. 2008; 29(12): 2243-51.

45. Barker SD, Coolidge CJ, Kanerva A, Hakkarainen T, Yamamoto M, Liu B, et al. The secretory leukoprotease inhibitor (SLPI) promoter for ovarian cancer gene therapy. J Gene Med. 2003; 5(4): 300-10.

46. Labidi-Galy SI, Clauss A, Ng V, Duraisamy S, Elias KM, Piao HY, et al. Elafin drives poor outcome in high-grade serous ovarian cancers and basal-like breast tumors. Oncogene. 2015; 34(3): 373-83.

47. Wei H, Hellstrom KE, Hellstrom I. Elafin selectively regulates the sensitivity of ovarian cancer cells to genotoxic drug-induced apoptosis. Gynecol Oncol. 2012; 125(3): 727-33.

48. Larsen M, Ressler SJ, Gerdes MJ, Lu B, Byron M, Lawrence JB, et al. The WFDC1 gene encoding ps20 localizes to $16 \mathrm{q} 24$, a region of $\mathrm{LOH}$ in multiple cancers. Mamm Genome. 2000; 11(9): 767-73.

49. Hickman OJ, Smith RA, Dasgupta P, Rao SN, Nayak S, Sreenivasan S, et al. Expression of two WFDC1/ps20 isoforms in prostate stromal cells induces paracrine apoptosis through regulation of PTGS2/COX-2. Br J Cancer. 2016; 114(11): 1235-42

50. Solis-Calero C, Carvalho HF. Phylogenetic, molecular evolution and structural analyses of the WFDC1/prostate stromal protein 20 (ps20). Gene. 2019; 686: 125-40.

51. Taylor DD, Gercel-Taylor C, Parker LP. Patient-derived tumor-reactive antibodies as diagnostic markers for ovarian cancer. Gynecol Oncol. 2009; 115(1): 112-20.

52. Bast RC Jr, Feeney M, Lazarus H, Nadler LM, Colvin RB, Knapp RC. Reactivity of a monoclonal antibody with human ovarian carcinoma. J Clin Invest. 1981; 68(5): 1331-7.

53. Niloff JM, Bast RC Jr, Schaetzl EM, Knapp RC. Predictive value of CA 125 antigen levels in second-look procedures for ovarian cancer. Am J Obstet Gynecol. 1985; 151(7): 981-6.

54. Yin BW, Lloyd KO. Molecular cloning of the CA125 ovarian cancer antigen: dentification as a new mucin, MUC16. J Biol Chem. 2001; 276(29): 27371-5.

55. Yuan Q, Song J, Yang W, Wang H, Huo Q, Yang J, et al. The effect of CA125 on metastasis of ovarian cancer: old marker new function. Oncotarget. 2017; 8(30): 50015-22.

56. Aithal A, Junker WM, Kshirsagar P, Das S, Kaur S, Orzechowski C, et al. Development and characterization of carboxy-terminus specific monoclonal antibodies for understanding MUC16 cleavage in human ovarian cancer. PLoS One. 2018; 13(4): e0193907.

57. Aithal A, Rauth S, Kshirsagar P, Shah A, Lakshmanan I, Junker WM, et al. MUC16 as a novel target for cancer therapy. Expert Opin Ther Targets. 2018; 22(8): 675-86.

58. Terry KL, Sluss PM, Skates SJ, Mok SC, Ye B, Vitonis AF, et al. Blood and urine markers for ovarian cancer: a comprehensive review. Dis Markers. 2004; 20(2): 53-70.

59. Jacobs IJ, Menon U, Ryan A, Gentry-Maharaj A, Burnell M, Kalsi JK, et al. Ovarian cancer screening and mortality in the UK Collaborative Trial of Ovarian Cancer Screening (UKCTOCS): a randomised controlled trial. Lancet. 2016; 387(10022): 945-56.

60. Lee SW, Lee HY, Bang HJ, Song HJ, Kong SW, Kim YM. An improved prediction model for ovarian cancer using urinary biomarkers and a novel validation strategy. Int J Mol Sci. 2019; 20(19):4938.

61. Galgano MT, Hampton GM, Frierson HF Jr. Comprehensive analysis of HE4 expression in normal and malignant human tissues. Mod Pathol. 2006; 19(6): 847-53.

62. Jia MM, Deng J, Cheng XL, Yan Z, Li OC, Xing YY, et al. Diagnostic accuracy of urine HE4 in patients with ovarian cancer: a meta-analysis. Oncotarget. 2017; 8(6): 9660-71

63. Liao JB, Yip YY, Swisher EM, Agnew K, Hellstrom KE, Hellstrom I. Detection of the HE4 protein in urine as a biomarker for ovarian neoplasms: Clinical correlates. Gynecol Oncol. 2015; 137(3): 430-5.

64. Nagy B Jr, Krasznai ZT, Balla H, Csoban M, Antal-Szalmas P, Hernadi Z, et al. Elevated human epididymis protein 4 concentrations in chronic kidney disease. Ann Clin Biochem. 2012; 49(Pt 4): 377-80.

65. Moore RG, Brown AK, Miller MC, Skates S, Allard WJ, Verch T, et al. The use of multiple novel tumor biomarkers for the detection of ovarian carcinoma in patients with a pelvic mass. Gynecol Oncol. 2008; 108(2): 402-8.

66. Hamed EO, Ahmed H, Sedeek OB, Mohammed AM, Abd-Alla AA, Abdel Ghaffar HM. Significance of HE4 estimation in comparison with CA125 in diagnosis of ovarian cancer and assessment of treatment response. Diagn Pathol. 2013; 8: 11

67. Anastasi E, Granato T, Falzarano R, Storelli P, Ticino A, Frati L, et al. The use of HE4, CA125 and CA72-4 biomarkers for differential diagnosis between ovarian endometrioma and epithelial ovarian cancer. J Ovarian Res. 2013; 6(1): 44 
68. Esselen KM, Cronin AM, Bixel K, Bookman MA, Burger RA, Cohn DE, et al. Use of CA-125 tests and computed tomographic scans for surveillance in ovarian cancer. JAMA Oncol. 2016; 2(11): 1427-33.

69. Vallius T, Hynninen J, Auranen A, Matomaki J, Oksa S, Roering P, et al. Postoperative human epididymis protein 4 predicts primary therapy outcome in advanced epithelial ovarian cancer. Tumour Biol. 2017; 39(2): 1010428317691189

70. Lycke M, Kristjansdottir B, Sundfeldt K. A multicenter clinical trial validating the performance of HE4, CA125, risk of ovarian malignancy algorithm and risk of malignancy index. Gynecol Oncol. 2018; 151(1): 159-65.

71. Wei SU, Li H, Zhang B. The diagnostic value of serum HE4 and CA-125 and ROMA index in ovarian cancer. Biomed Rep. 2016; 5(1): 41-4.

72. Kim B, Park Y, Kim B, Ahn HJ, Lee KA, Chung JE, et al. Diagnostic performance of CA 125, HE4, and risk of Ovarian Malignancy Algorithm for ovarian cancer. J Clin Lab Anal. 2019; 33(1): e22624.

73. Lakshmanan M, Kumar V, Chaturvedi A, Misra S, Gupta S, Akhtar N, et al. Role of serum HE4 as a prognostic marker in carcinoma of the ovary. Indian J Cancer. 2019; 56(3): 216-21.

74. Carlson AM, Maurer MJ, Goergen KM, Kalli KR, Erskine CL, Behrens MD, et al. Utility of progranulin and serum leukocyte protease inhibitor as diagnostic and prognostic biomarkers in ovarian cancer. Cancer Epidemiol Biomarkers Prev. 2013; 22(10): 1730-5.

75. Havrilesky LJ, Whitehead CM, Rubatt JM, Cheek RL, Groelke J, He Q, et al. Evaluation of biomarker panels for early stage ovarian cancer detection and monitoring for disease recurrence. Gynecol Oncol. 2008; 110(3): 374-82.

76. Han C, Bellone S, Siegel ER, Altwerger G, Menderes G, Bonazzoli E, et al. A novel multiple biomarker panel for the early detection of high-grade serous ovarian carcinoma. Gynecol Oncol. 2018; 149(3): 585-91.

77. Dochez V, Caillon H, Vaucel E, Dimet J, Winer N, Ducarme G. Biomarkers and algorithms for diagnosis of ovarian cancer: CA125, HE4, RMI and ROMA, a review. J Ovarian Res. 2019; 12(1): 28.

78. Bandiera E, Romani C, Specchia C, Zanotti L, Galli C, Ruggeri G, et al. Serum human epididymis protein 4 and risk for ovarian malignancy algorithm as new diagnostic and prognostic tools for epithelial ovarian cancer management. Cancer Epidemiol Biomarkers Prev. 2011; 20(12): 2496-506.

79. Zhang L, Chen Y, Wang K. Comparison of CA125, HE4, and ROMA index for ovarian cancer diagnosis. Curr Probl Cancer. 2019; 43(2): 135-44.

80. Chen F, Shen J, Wang J, Cai P, Huang Y. Clinical analysis of four serum tumor markers in 458 patients with ovarian tumors: diagnostic value of the combined use of HE4, CA125, CA19-9, and CEA in ovarian tumors. Cancer Manag Res. 2018; 10: 1313-8.

81. Kim YW, Bae SM, Lim H, Kim YJ, Ahn WS. Development of multiplexed bead-based immunoassays for the detection of early stage ovarian cancer using a combination of serum biomarkers. PLoS One. 2012; 7(9): e44960.

82. Casova M, Kucera R, Svobodova S, Presl J, Topolcan O, Novotny Z, et al. HE4 in comparison with other biomarkers in ovarian cancer diagnostics. PLZEN MEDICAL REPORT. 2014; 80 (2015) :21-26.

83. Zheng X, Chen S, Li L, Liu X, Liu X, Dai S, et al. Evaluation of HE4 and TTR for diagnosis of ovarian cancer: Comparison with CA-125. J Gynecol Obstet Hum Reprod. 2018; 47(6): 227-30.

84. Yurkovetsky Z, Skates S, Lomakin A, Nolen B, Pulsipher T, Modugno F, et al. Development of a multimarker assay for early detection of ovarian cancer. J Clin Oncol. 2010; 28(13): 2159-66.

85. Moore RG, Miller MC, Disilvestro P, Landrum LM, Gajewski W, Ball JJ, et al. Evaluation of the diagnostic accuracy of the risk of ovarian malignancy algorithm in women with a pelvic mass. Obstet Gynecol. 2011; 118(2 Pt 1): 280-8.

86. Coleman RL, Herzog TJ, Chan DW, Munroe DG, Pappas TC, Smith A, et al. Validation of a second-generation multivariate index assay for malignancy risk of adnexal masses. Am J Obstet Gynecol. 2016; 215(1): 82 e1- e11.

87. Urban RR, Pappas TC, Bullock RG, Munroe DG, Bonato V, Agnew K, et al. Combined symptom index and second-generation multivariate biomarker test for prediction of ovarian cancer in patients with an adnexal mass. Gynecol Oncol. 2018; 150(2): 318-23 